\title{
O Ensino de Algoritmos e Lógica de Programação como uma Ferramenta Pedagógica para Auxiliar a Aprendizagem de Matemática: Um Relato de Experiência
}

\author{
Claudivan Cruz Lopes ${ }^{1}$, Maria do Socorro dos Santos Guedes Duarte ${ }^{1}$, Enos \\ Andrade Diniz Sousa ${ }^{1}$, Rakel Pereira de Souza ${ }^{2}$, Isolda Bezerra Pereira ${ }^{2}$ \\ ${ }^{1}$ Instituto Federal de Educação, Ciência e Tecnologia da Paraíba (IFPB) \\ Campus Patos - Patos, Paraíba - Brasil \\ ${ }^{2}$ Universidade Estadual da Paraíba (UEPB) \\ Campus VII - Patos, Paraíba - Brasil \\ \{claudivan, maria.duarte\} @ifpb.edu.br, \\ \{enosads, rakelsouza7, isoldapereira2\} dgmail.com
}

\begin{abstract}
The learning of algorithms and programming logic (APL) is essential for the improvement of the logical reasoning and for the ability to solve problems. Nevertheless, the teaching of APL has been little exploited as a pedagogical tool that can help the learning of mathematics and improve the ability to solve math problems. This article reports an experience regarding the teaching of APL to 5th graders, whose focus was the creation of programs that applied mathematical concepts studied in the classroom. The results of this experience indicated that the teaching of APL can stimulate the interest as well as improve the understanding of mathematics.
\end{abstract}

Resumo. O aprendizado de algoritmos e lógica de programação (ALP) é fundamental para o desenvolvimento do raciocínio lógico e da capacidade de resolver problemas. Entretanto, o ensino de ALP tem sido pouco explorado como uma ferramenta pedagógica para auxiliar na aprendizagem de matemática e melhorar a capacidade de resolver problemas matemáticos. Este artigo relata uma experiência de ensino de ALP para alunos do $5^{\circ}$ ano do ensino fundamental, cujo enfoque foi na criação de programas que aplicaram os conceitos de matemáticos estudados em sala de aula. Os resultados obtidos nesta experiência indicaram que o ensino de ALP pode estimular o interesse pela matemática e melhorar a compreensão da matemática.

\section{Introdução}

No cenário da educação no Brasil existe uma clara dificuldade quanto ao aprendizado de conteúdos de matemática por parte dos alunos do ensino fundamental, onde um dos reflexos dessa dificuldade é o atraso no desenvolvimento do raciocínio lógico e na capacidade de resolver problemas (SANTOS; BASSO, 2012; ANDRADE et al., 2013).

Como tentativa de minimizar ou suprimir esta dificuldade, diversas iniciativas pedagógicas têm surgido no intuito de auxiliar a aprendizagem de matemática, em particular, a iniciativa da aprendizagem apoiada pelo uso de ferramentas educacionais computadorizadas, tais como programas educacionais, jogos e robótica educacional 
V Congresso Brasileiro de Informática na Educação (CBIE 2016)

Anais do XXII Workshop de Informática na Escola (WIE 2016)

(ZORZAN, 2007; IASCHVISTIK; BRANDALISE, 2011; GALLEGO, 2007). O uso de ferramentas educacionais computadorizadas contribui como fator motivacional para estimular a aprendizagem, e permite que uma criança desenvolva suas habilidades em matemática a partir do aspecto lúdico-didático intrínseco nestas ferramentas, onde assim, é possível aprender matemática brincando (MARTINELLI, 2014).

Por outro lado, em várias partes do mundo, algoritmos e lógica de programação (ALP) estão sendo ensinados desde os primeiros níveis da educação formal (NCE, 2015; $\mathrm{K} 12$, 2015), onde estudos indicam que a inclusão do pensamento algorítmico numa criança é fundamental para seu crescimento intelectual, pois ela ganha habilidades mentais para toda a vida, treinadas pela programação, e que a prepara para a resolução de problemas em diversas áreas do conhecimento humano (WANGENHEIM et al., 2014; RESNICK et al., 2009; RAMOS; TEIXEIRA, 2015).

Considerando o contexto em que ferramentas educacionais computadorizadas têm se tornado importantes instrumentos pedagógicos no auxílio da aprendizagem da matemática, e que estudos apontam que o ensino de ALP tem sido útil para o desenvolvimento do raciocínio lógico de uma criança (WING, 2006; MOTA et al., 2014), então uma equipe multidisciplinar propôs uma unidade instrucional (UI) para o ensino de ALP para alunos do ensino fundamental, cujo enfoque é dado no exercício de conteúdos de matemática. Uma unidade instrucional (UI) é um conjunto de lições sobre um tema de estudo, e é organizada numa sequencia incremental de desenvolvimento de habilidades relacionadas ao tema estudado (DAY; HURWITZ, 2011).

Em geral, as metas introduzidas com a proposição desta UI foram de natureza dual: primeiro, pretendeu-se estimular o pensamento algorítmico e o raciocínio lógico exigido na resolução de problemas; e segundo, objetivou-se incentivar a admiração pela matemática e auxiliar a aprendizagem da matemática a partir da aprendizagem de ALP com uso de uma ferramenta educacional computadorizada. Para cumprir estas metas, a UI foi planejada de maneira que os conteúdos de matemática estudados em sala de aula fossem aplicados como exercícios nas práticas de ALP. Desse modo, verificou-se que os conteúdos de matemática vistos em sala de aula foram revisados e treinados a partir da prática de programação de computadores, onde assim, foi possível aprender matemática programando.

Neste artigo é relatada uma experiência positiva em relação aos resultados alcançados com a execução da UI proposta. Esta experiência foi realizada numa escola estadual de ensino fundamental, onde o público-alvo foi formado por alunos do $5^{\circ}$ ano. Os resultados obtidos com a execução desta experiência indicaram que a metodologia proposta na UI viabilizou o suporte ao aprendizado de matemática. Esse resultado foi constatado por meio de depoimentos dos professores de matemática envolvidos nesta experiência, e também por resultados quantitativos colhidos durante a realização desta experiência, demonstrando que é possível auxiliar a aprendizagem de matemática a partir do ensino de ALP.

O restante deste artigo está organizado nas seções a seguir. A Seção 2 descreve um modelo de UI para o ensino de ALP com foco em matemática. A Seção 3 relata uma experiência de execução de uma UI baseada neste modelo. A Seção 4 discute resultados desta experiência, enquanto a Seção 5 conclui o artigo e apresenta trabalhos futuros. 
V Congresso Brasileiro de Informática na Educação (CBIE 2016)

Anais do XXII Workshop de Informática na Escola (WIE 2016)

\section{Um Modelo de UI para o Ensino de ALP com Foco em Matemática}

Para nortear a elaboração deste modelo de UI, a seguinte hipótese foi levantada: como é possível apoiar a aprendizagem de conteúdos de matemática a partir do ensino de ALP? $\mathrm{Na}$ investigação dessa hipótese, um requisito fundamental foi considerado: o ensino de ALP deve atuar como uma ferramenta motivacional e facilitadora para a aprendizagem de matemática. Desse modo, tendo a hipótese e o requisito em vista, a primeira etapa no desenvolvimento deste modelo de UI foi delimitar seu escopo, onde foram definidos os objetivos geral e específicos, público-alvo e o contexto de aplicação da UI. Em seguida, foi planejada a metodologia de trabalho para guiar uma execução da UI.

\subsection{Objetivos}

O objetivo geral é propor diretrizes metodológicas para o desenvolvimento de uma UI para apoiar o processo de aprendizagem de conteúdos de matemática estudados no ensino fundamental, a partir do ensino de ALP com enfoque na resolução de problemas matemáticos implementados como programas de computador de baixa complexidade.

Para alcançar o objetivo geral, são definidos os seguintes objetivos específicos: estimular o raciocínio lógico através do pensamento algorítmico; introduzir conceitos de ALP; entender a importância do pensamento algorítmico na resolução de problemas matemáticos; incentivar o aprendizado da matemática e estimular a admiração e o gosto pelo estudo da matemática; auxiliar o aprendizado de matemática a partir da criação de algoritmos e programas que apliquem os conceitos de matemática estudados em sala de aula; e auxiliar o processo de ensino e aprendizagem de matemática a partir do uso de uma ferramenta pedagógica computacional para o ensino de ALP.

\subsection{Público-alvo e Contexto}

Quanto ao público-alvo, a UI deve ser projetada para ser aplicada com crianças na faixa etária de 10 a 14 anos, entre o $5^{\circ}$ ano e o $9^{\circ}$ ano do ensino fundamental. As crianças devem possuir conhecimentos básicos de informática e experiência na utilização de computadores. Tipicamente, estes conhecimento e experiência refletem as interações das crianças com jogos educacionais, jogos de entretenimento, navegação na $W e b$, digitação de textos curtos e manuseio de sistemas operacionais e de programas pouco sofisticados para editoração de imagens. Além disso, e por consequência das experiências adquiridas com estas interações, as crianças devem dominar a operação básica de um computador, como ligar e desligar, e a operação de dispositivos de hardware como teclado, mouse e monitor. Também se deve encorajar a participação de toda criança interessada na UI, sem qualquer restrição de seleção em relação ao seu grau de habilidades sobre ALP. O mesmo é verdadeiro quanto ao nível de afinidade e de habilidades adquiridas em relação aos conteúdos de matemática vistos nas suas séries, uma vez que o intercâmbio de conhecimento entre indivíduos com níveis cognitivos distintos é de suma importância para se alcançar uma aprendizagem coletiva satisfatória (REATEGUI; NOTARE, 2004).

Quanto ao contexto de aplicação da UI, esta deve ser projetada para ser aplicada em escolas de ensino fundamental que disponham de laboratório de informática para a realização das aulas práticas de programação em horário oposto, de modo a não prejudicar o andamento das aulas regulares de um ano letivo. Não deve haver restrições quanto ao sistema operacional utilizado nos computadores do laboratório, porém deve 
haver a disponibilidade de uso de um computador por criança, e as crianças devem estar familiarizadas com o manuseio desse sistema operacional. O laboratório deve estar em pleno funcionamento e apresentar conforto térmico, e deve ser equipado com projetor multimídia ou lousa digital, e quadro branco e pincel. Além disso, o apoio logístico da escola é fundamental para uma condução satisfatória de execução da UI, assim como é essencial o engajamento dos professores de matemática envolvidos no desenvolvimento da UI, desde o planejamento até sua concreta execução.

\subsection{Metodologia}

A metodologia adotada para o desenvolvimento da UI deve compreender as seguintes etapas: (I) definição de ementas e conteúdos de ALP; (II) elaboração de um plano de aulas; (III) identificação do conjunto de conteúdos de matemática ensinados em sala de aula, e sua respectiva distribuição no plano de aulas; (IV) confecção de material didático e exercícios; (V) cadastramento, triagem e identificação de perfis de alunos quanto ao grau de dificuldade em matemática; e (VI) execução e registro de aulas e de atividades.

A concepção das ementas deve ser baseada em conteúdos comumente lecionados nas disciplinas introdutórias de ALP de cursos de ciência da computação. Portanto, é sugerido o seguinte ementário: introdução aos algoritmos e lógica de programação; ferramentas pedagógicas computacionais para o ensino de ALP; resolução de problemas usando algoritmos e raciocínio lógico; tipos de dados, variáveis e constantes; expressões e operadores lógicos e aritméticos; estruturas condicionais e estruturas de repetição. Este ementário pode ser distribuído em conteúdos programáticos dividido em três unidades, conforme a Figura 1. A unidade I destina-se ao entendimento de aspectos introdutórios de ALP, incluindo o manuseio de uma ferramenta educacional computadorizada para o ensino de ALP. A unidade II destina-se ao desenvolvimento de habilidades relacionadas aos conteúdos específicos de ALP. Já a unidade III enfatiza na iniciação à criação de jogos educacionais matemáticos, onde podem ser praticados conteúdos de matemática e ALP estudados durante a realização da UI. Desse modo, pretende-se consolidar o prazer pela programação de computadores e especialmente pelo estudo da matemática.

\begin{tabular}{|c|c|c|}
\hline I - Introdução & II - Lógica de Programação & $\begin{array}{c}\text { III - Jogos Matemáticos } \\
\text { Educacionais }\end{array}$ \\
\hline $\begin{array}{l}\text { - Resolvendo problemas no mundo } \\
\text { real com algoritmos } \\
\text { - Introdução aos algoritmos e lógica } \\
\text { de programação } \\
\text { - Representação de algoritmos } \\
\text { - Introdução a uma ferramenta } \\
\text { educacional computadorizada para } \\
\text { o ensino de algoritmos }\end{array}$ & $\begin{array}{l}\text { - Saída de dados } \\
\text { - Variáveis, constantes e tipos de dados } \\
\text { - Atribuição } \\
\text { - Leitura de dados } \\
\text { - Operadores e expressões aritméticas } \\
\text { - Operadores lógicos e relacionais } \\
\text { - Comandos de decisão } \\
\text { - Comandos de repeticão }\end{array}$ & $\begin{array}{l}\text { - Introdução aos jogos } \\
\text { educativos } \\
\text { - Definição de objetivos dos } \\
\text { jogos } \\
\text { - Cenários e personagens } \\
\text { - Critérios de pontuação } \\
\text { - Prototipação e testes }\end{array}$ \\
\hline
\end{tabular}

Figura 1. Proposta de conteúdo programático de ALP

As etapas II e III (i.e. plano de aulas e identificação e distribuição de conteúdos de matemática) devem ser planejadas em reuniões que incluam a equipe multidisciplinar responsável pela UI e também os professores de matemática das séries envolvidas na execução da UI, uma vez que o objetivo da UI é auxiliar a aprendizagem de matemática a partir do ensino de ALP. Com o auxilio destes professores, a equipe multidisciplinar será capaz de definir como os conteúdos de matemática vistos em sala de aula deverão ser revisados e exercitados por meio da criação de programas de computadores durante as aulas de ALP. Para orientar o planejamento destas etapas, foi criado um formulário- 
V Congresso Brasileiro de Informática na Educação (CBIE 2016)

Anais do XXII Workshop de Informática na Escola (WIE 2016)

modelo, conforme ilustrado na Figura 2, onde é possível enumerar a aula e determinar seus objetivos, assim como correlacionar os conteúdos de matemática aos conteúdos de ALP que devem ser praticados em cada aula. Um exemplo de um planejamento de aulas executado numa experiência-piloto é apresentado na Seção 3.

\begin{tabular}{|c|c|c|c|}
\hline \multirow{2}{*}{$\#$} & \multirow{2}{*}{ Objetivos } & \multicolumn{2}{|c|}{ Conteúdos Estudados } \\
\cline { 3 - 4 } & & Matemática & ALP \\
\hline & & & \\
\hline
\end{tabular}

Figura 2. Modelo de plano de aulas

Quanto à elaboração dos materiais didáticos usados nas aulas de ALP (i.e. etapa IV da metodologia proposta), estes devem apresentar o título do conteúdo de ALP a ser abordado na aula, e a indicação dos conteúdos de matemática que serão praticados, além de exemplos ilustrativos dos conteúdos de ALP criados a partir de exemplos retirados do livro didático adotado pelos professores de matemática. Os materiais devem priorizar o exercício prática dos conteúdos de ALP e de matemática. Recomenda-se também que os tópicos introdutórios de cada material de aula apresentem uma revisão sintética dos conteúdos de matemática a serem abordados nas aulas de ALP. Uma orientação quanto à formatação dos materiais didáticos é que estes procurem manter o padrão de cores e fontes dos livros didáticos de matemática adotados. De maneira complementar, as listas de exercícios de ALP devem ser baseadas nos exercícios propostos no livro didático e nos exercícios propostos pelos professores de matemática durante suas aulas.

A próxima etapa é realizar um cadastro e triagem dos alunos envolvidos na execução da UI, e identificar os perfis de alunos em relação ao grau de dificuldade em matemática (i.e. etapa V). O cadastramento deve conter dados pessoais e demográficos, enquanto a triagem e a identificação de perfis devem ser úteis para fins de avaliação dos resultados da experiência. Sugere-se uma classificação de alunos nos perfis muita dificuldade, dificuldade moderada e pouca dificuldade em matemática. Os resultados dessa classificação podem ser obtidos por meio de entrevistas com os professores de matemática e pelos resultados acadêmicos colhidos até o início da execução da UI.

A última etapa da metodologia proposta (i.e. etapa VI) corresponde à execução da UI efetivamente. As aulas ministradas devem ser registradas em formulário próprio, indicando data/hora da aula, conteúdo ministrado e assinatura dos instrutores. O registro de atividades e de avaliação da aprendizagem deve ser feito continuamente a partir da aplicação de exercícios individuais e em grupos, e da criação de um jogo matemático educacional, conforme a unidade III do conteúdo programático. Também, os resultados das avaliações da disciplina Matemática feitas durante a realização da UI devem ser obtidos juntamente com as observações dos professores de matemática em relação ao desempenho dos alunos. Desse modo, é possível apurar os resultados da UI e produzir uma conclusão que descreva a evolução da aprendizagem de matemática e de ALP.

\section{Um Relato de Experiência}

Com base no modelo proposto na Seção 2, foi planejada e desenvolvida uma UI para o ensino de ALP com foco em conteúdos de matemática para alunos do $5^{\circ}$ ano do ensino fundamental da Escola Estadual de Ensino Fundamental e Médio Rio Branco, situada na cidade de Patos-PB. O desenvolvimento desta UI foi fruto de um projeto de extensão 
V Congresso Brasileiro de Informática na Educação (CBIE 2016)

Anais do XXII Workshop de Informática na Escola (WIE 2016)

que visou introduzir o pensamento algorítmico em crianças entre 9 e 11 anos e avaliar o impacto do ensino de ALP nos resultados acadêmicos da disciplina Matemática.

A equipe envolvida neste projeto foi composta por dois professores de ciência da computação, dois professores de matemática, um pedagogo, dois alunos de ciência da computação e um aluno de curso técnico em informática. $\mathrm{O}$ início dos trabalhos ocorreu no começo de maio de 2015, onde a equipe realizou reuniões para delimitar as ementas e conteúdos relacionados ao ensino de ALP. Assim, foi produzido o ementário da Seção 2.3 e os conteúdos programáticos ilustrados na Figura 1. Paralelamente, as condições de uso do laboratório de informática cedido pela escola foram avaliadas, o qual dispunha de vinte computadores, ar condicionado e projeto multimídia.

Em seguida, reuniões de planejamento foram feitas para delinear o escopo da UI quanto aos conteúdos de matemática do $5^{\circ}$ ano fundamental que deveriam ser treinados nas aulas de ALP. Foi decidido que as aulas introdutórias de ALP deveriam envolver alguns conteúdos de matemática estudados no primeiro semestre do ano letivo 2015, e que os conteúdos de matemática ministrados em sala de aula no segundo semestre (i.e. no decorrer da execução da UI) seriam revisados e praticados nas aulas de ALP somente a partir do segundo mês de execução da UI. A Figura 3 ilustra uma parte do plano de aulas produzido, utilizando o formato do formulário-modelo exibido na Figura 2.

\begin{tabular}{|c|c|c|c|}
\hline \multirow{2}{*}{ \# } & \multirow{2}{*}{ Objetivos } & \multicolumn{2}{|c|}{ Conteúdos Estudados } \\
\hline & & Matemática & ALP \\
\hline 03 & $\begin{array}{l}\text { - Conhecer operadores e } \\
\text { expressões aritméticas. }\end{array}$ & $\begin{array}{l}\text { - Números; } \\
\text { • Adição, subtração, } \\
\text { multiplicação e divisão. }\end{array}$ & $\begin{array}{l}\text { - Variáveis e tipos de dados numéricos; } \\
\text { - Comando de atribuição; } \\
\text { - Leitura de dados; } \\
\text { - Operadores e expressões aritméticas. }\end{array}$ \\
\hline 04 & $\begin{array}{l}\text { - Conhecer e diferenciar formas } \\
\text { geométricas; } \\
\text { - Calcular áreas e perímetros; } \\
\text { - Realizar orientação especial no } \\
\text { plano cartesiano. }\end{array}$ & $\begin{array}{l}\text { - Formas geométricas planas; } \\
\text { - Formas geométricas } \\
\text { espaciais. }\end{array}$ & $\begin{array}{l}\text { - Variáveis e tipos de dados numéricos; } \\
\text { - Comando de atribuição; } \\
\text { - Leitura de dados; } \\
\text { - Operadores e expressões aritméticos; } \\
\text { - Operadores e expressões lógicos; } \\
\text { - Estruturas de decisão. }\end{array}$ \\
\hline 09 & $\begin{array}{l}\text { - Conhecer noções básicas de } \\
\text { retas, ângulos e circunferência } \\
\text { por meio das animações no } \\
\text { Scratch. }\end{array}$ & $\begin{array}{l}\text { - Retas; } \\
\text { - Ângulos; } \\
\text { - Circunferência. }\end{array}$ & $\begin{array}{l}\text { - Entrada e saída de dados; } \\
\text { - Variáveis e tipos de dados numéricos; } \\
\text { - Comando de atribuição; } \\
\text { - Operadores e expressões aritméticas. }\end{array}$ \\
\hline 15 & $\begin{array}{l}\text { - Desenvolvimento de um jogo } \\
\text { matemático usando o Scratch } \\
\text { (Personagens e animações). }\end{array}$ & $\begin{array}{l}\text { - Qualquer conteúdo de } \\
\text { matemática ministrado no } 5^{\circ} \\
\text { ano. }\end{array}$ & $\begin{array}{l}\text { - Variáveis e tipos de dados numéricos; } \\
\text { - Comando de atribuição; } \\
\text { - Leitura de dados; } \\
\text { - Operadores e expressões aritméticas; } \\
\text { - Operadores e expressões lógicas; } \\
\text { - Estruturas de decisão e de repetição. }\end{array}$ \\
\hline
\end{tabular}

Figura 3. Trecho do plano de aulas produzido

Com a concepção do plano de aulas efetuada, a equipe realizou a produção do material didático, e o cadastro, triagem e identificação de perfis de alunos. Foi obtido um exemplar do livro-texto de matemática adotado no $5^{\circ}$ ano da escola Rio Branco para auxiliar na confecção dos materiais e exercícios. Além de seguir as diretrizes da Seção 2.3 para a produção de materiais didáticos, foi feita a escolha da ferramenta educacional computadorizada para o ensino de ALP, já que os materiais deveriam se basear no uso de uma ferramenta especifica. A ferramenta escolhida foi o Scratch (RESNICK et al., 2009), devido a sua ampla popularização e a familiarização dos membros da equipe quanto ao seu manuseio. A Figura 4 ilustra um exemplo de slides feitos para a aula 03 (conforme a Figura 3), os quais apresentam uma revisão dos conteúdos de matemática praticados na aula, e um exemplo de um programa Scratch para somar dois números. 
Quanto ao cadastramento, foi confeccionada uma ficha de inscrição para que os alunos participantes da UI pudessem informar seus dados pessoais e demográficos. A anuência dos responsáveis do aluno quanto à participação na UI também foi necessária, já que sua execução foi prevista para horário oposto, fato que gerou deslocamento dos alunos para a escola fora do horário regular de aulas.

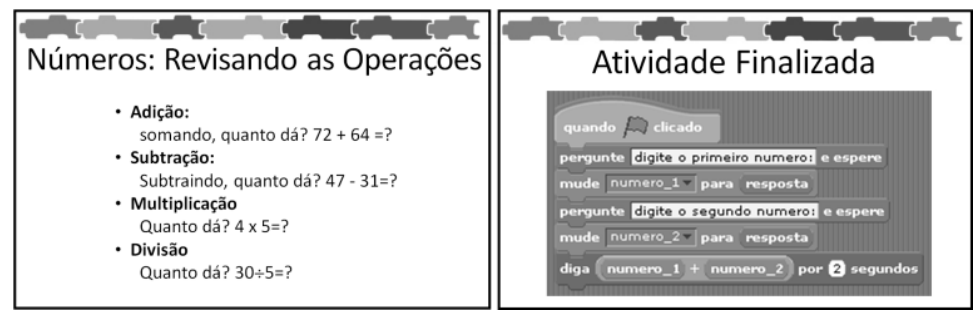

Figura 4. Exemplo de material didático produzido

Quanto à triagem e identificação de perfis de aluno, foram feitas entrevistas com os professores de matemática do $5^{\circ}$ ano e foram colhidos os resultados acadêmicos de matemática dos alunos participantes da UI no primeiro semestre letivo, i.e. de fevereiro à junho de 2015. A escola adota um sistema de avaliação baseado em conceito, onde um aluno pode ser conceituado como $N$ (não adquiriu uma competência), $E P$ (em processo) e $S$ (adquiriu uma competência), em cada mês letivo cursado, para cada disciplina, e de acordo com algum critério de avaliação. Para a disciplina Matemática, a escola adota vinte critérios distintos. Este sistema facilitou a triagem e a identificação de perfis: $30 \%$ dos alunos inscritos foram classificados como N, 55\% EP e $15 \% \mathrm{~S}$, considerando um total de 40 inscrições e a medida de moda dos conceitos obtidos entre fevereiro e junho de 2015 nos vinte critérios pré-estabelecidos pela escola para a disciplina Matemática. Então, uma correlação foi feita entre os níveis de muita dificuldade em matemática para $\mathrm{N}$, de dificuldade moderada para EP e de pouca dificuldade para S. Assim, 30\% dos alunos inscritos estavam com muita dificuldade em matemática, 55\% com dificuldade moderada e $15 \%$ com pouca dificuldade em matemática antes do início da UI.

O início da execução da UI ocorreu em meados de agosto de 2015 e finalizou em meados de dezembro de 2015. Os 40 alunos inscritos foram divididos ao acaso em duas turmas de 20 alunos. Cada turma teve dois encontros semanais de $1 \mathrm{~h} 15 \mathrm{~min}$, totalizando uma carga horária de $48 \mathrm{~h}$ por turma. Um dos encontros semanais foi destinado à prática de exercícios, e os cinco últimos encontros foram reservados para o desenvolvimento de jogos educacionais matemáticos que abordaram conteúdos de matemática estudados no ano letivo, como operações aritméticas, números decimais e figuras geométricas planas. Reuniões quinzenais foram feitas entre a equipe e os professores de matemática do $5^{\circ}$ ano envolvidos nesta UI, a fim de averiguar o andamento da UI e de validar os registros de aulas realizadas e os conteúdos abordados. Estas reuniões também foram usadas para fazer avaliações subjetivas do grau de satisfação dos alunos em relação à UI proposta.

\section{Resultados e Discussão}

Durante o período de execução da UI, houve a desistência de 14 alunos. Assim, no total, 26 alunos concluíram e foram certificados. Os conceitos obtidos em Matemática no $1^{\circ}$ semestre de 2015 foram novamente tabulados para refletir apenas o grau de dificuldade em matemática dos alunos concluintes. Também foram colhidos os conceitos obtidos nos $3^{\circ}$ e $4^{\circ}$ bimestres (i.e. de agosto a dezembro de 2015), de modo que fosse possível 
V Congresso Brasileiro de Informática na Educação (CBIE 2016)

Anais do XXII Workshop de Informática na Escola (WIE 2016)

avaliar o impacto quantitativo do ensino de ALP na aprendizagem de matemática desses alunos. A Figura 5 ilustra o resultado final, admitindo o uso da medida de moda sobre os conceitos registrados para os vinte critérios de avalição de Matemática no $1^{\circ}$ semestre e nos $3^{\circ}$ e $4^{\circ}$ bimestres, e a correlação dos conceitos pré-definidos pela escola (i.e. S, EP e N) para os perfis de alunos adotados na UI (conforme descrito da Seção 3).

\begin{tabular}{|c|c|c|c|}
\hline Perfil & $\mathbf{1}^{\circ}$ Semestre & $\mathbf{3}^{\circ}$ Bimestre & $\mathbf{4}^{\circ}$ Bimestre \\
\hline Muita dificuldade & $27 \%$ & $20 \%$ & $23 \%$ \\
\hline Dificuldade moderada & $62 \%$ & $42 \%$ & $50 \%$ \\
\hline Pouca dificuldade & $11 \%$ & $38 \%$ & $27 \%$ \\
\hline
\end{tabular}

Figura 5. Resultados quantitativos obtidos

Analisando os resultados da Figura 5, percebe-se que o número de alunos com muita dificuldade em matemática diminuiu de $27 \%$ no $1^{\circ}$ semestre, para $20 \%$ e $23 \%$ nos $3^{\circ}$ e $4^{\circ}$ bimestres, respectivamente. Também houve uma redução no número de alunos com dificuldade moderada: diminuiu em $20 \%$ no $3^{\circ}$ bimestre em relação ao $1^{\circ}$ semestre, e em $12 \%$ no $4^{\circ}$ bimestre em comparação ao $1^{\circ}$ semestre. Em contrapartida, o número de alunos com pouca dificuldade em matemática aumentou de $11 \%$ no $1^{\circ}$ semestre para $38 \%$ e $27 \%$ nos $3^{\circ}$ e $4^{\circ}$ bimestres, respectivamente. Isso pressupôs a comprovação da hipótese de que o ensino de ALP pode auxiliar na aprendizagem de matemática. Porém, isto só foi confirmada a partir de uma avaliação qualitativa em relação à UI proposta.

A avaliação qualitativa foi realizada por meio da aplicação de questionários para os professores de matemática do $5^{\circ}$ ano envolvidos na UI e para os próprios alunos. $\mathrm{O}$ questionário dos professores foi formado por questões de respostas subjetivas quanto à percepção da evolução da aprendizagem dos alunos em matemática após a execução da UI; percepção quanto à satisfação dos alunos em relação à UI; percepção da execução da UI quanto aos objetivos, metodologia e planejamento de aulas; e proposta de melhorias. Suas respostas foram derivadas de observações feitas em sala de aula. Eles afirmaram que os alunos participantes da UI ficaram mais envolvidos nos conteúdos de matemática nos $3^{\circ}$ e $4^{\circ}$ bimestres e que a UI contribuiu para esse envolvimento. Também relataram o entusiasmo dos alunos por ALP, onde alguns deles disseram ter despertado o interesse por fazer uma faculdade de ciência da computação no futuro. Comentaram ainda que os alunos aprenderam a resolver problemas de matemática através de novos caminhos da tecnologia, e que os objetivos traçados na UI foram alcançados, que a metodologia executada foi de ótima clareza e o plano de aulas esteve em sintonia com os conteúdos de matemática apresentados em sala de aula. Sugeriram que a execução da UI atingisse um número maior de alunos e que fosse executada $2 \mathrm{x}$ ao ano. Também sugeriram que fosse ministrada uma capacitação para os professores quanto aos conteúdos de ALP.

Em relação ao questionário para os alunos, este permitiu conhecer suas opiniões quanto ao entusiasmo pela matemática, a satisfação em relação à UI, e os conteúdos de ALP que mais se identificaram. A Figura 6(a) apresenta os resultados do entusiasmo em matemática e da satisfação em relação à UI. Neste resultado, é importante destacar que o percentual de alunos que não gostavam de matemática diminuiu de $27 \%$ antes da UI, para $20 \%$ depois da execução da UI; e que $95 \%$ dos alunos aprovaram a execução da UI (i.e. 50\% Bom $+45 \%$ Excelente). Em relação aos conteúdos de ALP, 20\% e 27\% dos alunos preferiram lógica de programação e recursos de animação, respectivamente. Já $53 \%$ dos alunos preferiram a criação dos jogos educacionais matemáticos, conforme ilustra a Figura 6(b). Então, os alunos foram entrevistados para se entender este grau de 
satisfação em relação ao desenvolvimento dos jogos educacionais. Em geral, relataram que as aulas destinadas a esta finalidade foram um momento empolgante, onde puderam praticar a matemática e aprender sobre a criação de jogos. A Figura 7 ilustra exemplos de jogos desenvolvidos pelos alunos. A Figura 7(a) ilustra o jogo O Mundo Encantado da Matemática, cujo objetivo é resolver expressões aritméticas, e a Figura 7(b) apresenta o jogo Geometria, o qual exercita os conhecimentos sobre figuras geométricas planas.

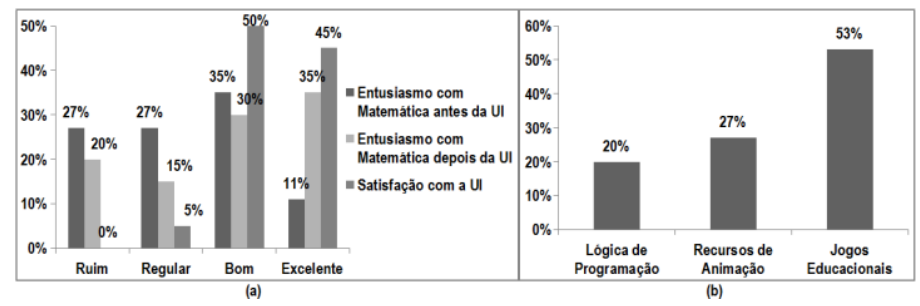

Figura 6. Resultados do questionário dos alunos

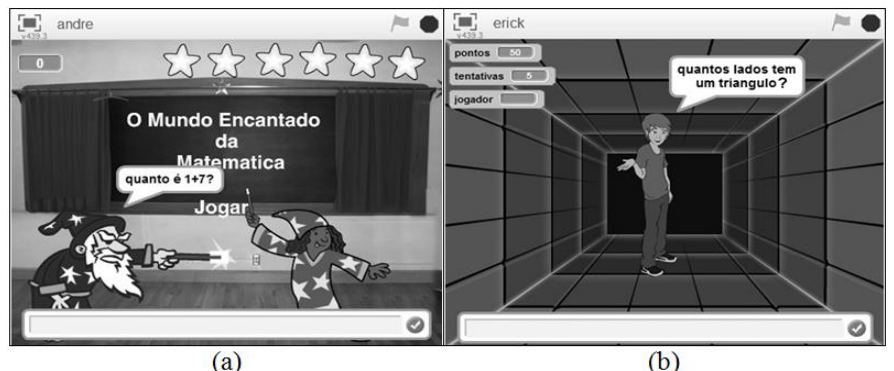

Figura 7. Jogos desenvolvidos pelos alunos durante a execução da UI

\section{Conclusão}

Este artigo relatou uma experiência do ensino de algoritmos e lógica de programação (ALP) como uma ferramenta pedagógica para auxiliar a aprendizagem de matemática. Esta experiência foi feita com alunos do $5^{\circ}$ ano do ensino fundamental, e foi baseada numa proposta metodológica que permitiu que os alunos pudessem estudar os conteúdos de matemática vistos em sala de aula de forma associada aos conteúdos de ALP. Como resultados desta experiência, houve uma redução de $7 \%$ e de $20 \%$ do número de alunos com muita dificuldade e com dificuldade moderada em matemática, respectivamente, e mais alunos passaram a ter um melhor entendimento dos assuntos de matemática, pois houve um aumento de $16 \%$ no número de alunos com pouca dificuldade em matemática. Também houve um índice de 95\% de satisfação dos alunos com esta experiência. Estes resultados foram validados qualitativamente com análises feitas sobre as respostas de questionários aplicados aos professores envolvidos nesta experiência, que indicaram que os alunos incluídos na experiência apresentaram melhoria no rendimento acadêmico de matemática e melhoria na capacidade de resolver de problemas matemáticos.

Como continuação desta experiência, pretende-se aplicar a metodologia proposta na execução de um curso de ALP em outras séries do ensino fundamental. Também será ofertado um treinamento para professores de matemática do ensino fundamental quanto à metodologia proposta, e um treinamento de ALP com o uso de ferramenta educacional computadorizada para o ensino de ALP. Assim, os professores podem autonomamente fazer uso sistemático do ensino de ALP como uma ferramenta pedagógica para auxiliar a aprendizagem de matemática. 
V Congresso Brasileiro de Informática na Educação (CBIE 2016)

Anais do XXII Workshop de Informática na Escola (WIE 2016)

\section{Referências}

ANDRADE, M.; SILVA, C.; OLIVEIRA, T. Desenvolvendo Games e Aprendendo Matemática Utilizando o Scratch. XII Simpósio Brasileiro de Jogos e Entreteinimento Digital, São Paulo, 2013. 260-263.

DAY, M.; HURWITZ, A. Children and Their Art: art Education for Elementary and Middle Schools. 9. ed. Boston: Wadsworth Publishing, 2011.

GALLEGO, J. P. A Utilização dos Jogos como Recurso Didático no EnsinoAprendizagem da Matemática. Faculdade de Ciências. Bauru, p. 80. 2007.

IASCHVISTIK, A. B. M.; BRANDALISE, M. A. T. Análise de Dados no Ensino Médio \& Tecnologia Educacional: uma Combinação Pedagógica Necessária. XIII Conferência Interamericana de Educação Matemática, Recife, 2011. 1-12.

K12. CSTA K-12 Computer Science Standards: Revised 2011. Disponível em: < http://www.csta.acm.org/Curriculum/sub/CurrFiles/CSTA_K-12_CSS.pdf $>$. Acesso em: 14 dez. 2015.

MARTINELLI, S. R. O Projeto Scratch Brasil: uma Iniciativa em Prol da Informática Educativa. Instituto Itapetiningano de Ensino Superior. Itapetininga, p. 142. 2014.

MOTA, F. P.; RIBEIRO, N. F.A.; EMMENDORFER, L.; BUTZEN, P.; MACHADO, K. S.; ADAMATTI, D. F. Desenvolvendo o Raciocínio Lógico no Ensino Médio: uma proposta utilizando a ferramenta Scratch. XXV Simpósio Brasileiro de Informática na Educação, Dourados, 2014, 377-381.

NCE. National curriculum in England: English Programmes of Study, 2013. Disponível em: < https://www.gov.uk/government/publications/national-curriculum-in-englandenglish-programmes-of-study>. Acesso em: 14 dez. 2015.

RAMOS, F.; TEIXEIRA, L. S. Significação da Aprendizagem Através do Pensamento Computacional no Ensino Médio: uma Experiência com Scratch. XXI Workshop de Informática na Escola, Maceió, 2015. 217-226.

REATEGUI, E.; NOTARE, M. A3: Ambiente de Aprendizagem de Algoritmos. XV Simpósio Brasileiro de Informática na Educação, Manaus, 2004, 1-3.

RESNICK, M. et al. Scratch Programming for All. Communications of the ACM, v. 52, n. 11, p. 60-67, 2009.

SANTOS, R. D. S.; BASSO, M. V. D. A. Tecnologias Digitais na Sala de Aula para Aprendizagem de Conceitos de Geometria Analítica: manipulações no Software GRAFEQ. A Matemática na Escola, n. 1, p. 177-196, 2012.

WANGENHEIM, C. G. V.; NUNES, V. R.; SANTOS, G. D. Ensino de Computação com Scratch no Ensino Fundamental: um Estudo de Caso. Revista Brasileira de Informática na Educação, v. 22, n. 3, p. 115-125, 2014.

WING, J. M. Computational Thinking. Communications of the ACM, v. 49, n. 3, p. 33$35,2006$.

ZORZAN, A. S. L. Ensino-Aprendizagem: algumas Tendências na Educação Matemática. Revista de Ciências Humanas - Educação, v. 8, n. 10, p. 77-94, 2007. 